\title{
Estimation of 2- and 3-parameter Burr Type XII distributions using EM algorithm
}

\author{
Nor Hidayah Ismail* and Zarina Mohd Khalid \\ Department of Mathematical Sciences, Faculty of Science, Universiti Teknologi Malaysia 81310 Skudai \\ *Corresponding Author: nhidayah268@gmail.com (N.H. Ismail)
}

Article history :

Received 4 January 2014

Revised 24 March 2014

Accepted 1 April 2014

Available online 1 June 2014

\section{GRAPHICAL ABSTRACT}

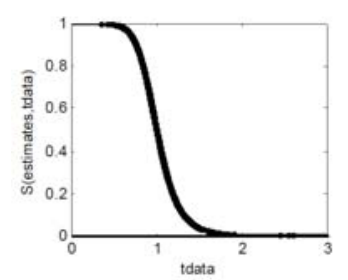

\begin{abstract}
The Burr Type XII distribution is one of the systems of continuous distributions and is widely known because the distribution includes the characteristics of various well known distributions such as Weibull and gamma distributions. Maximum likelihood estimation (MLE) has been a common method in estimating model parameters. An alternative method that is the expectation-maximization (EM) algorithm is presented in this paper to estimate the two- and three-parameter Burr Type XII distributions in the presence of complete and censored data. Furthermore, simulation study is conducted to compare the efficiency and accuracy of MLE and EM algorithm approaches. The result indicates that EM algorithm is more efficient and accurate than those estimates obtained via MLE approach.
\end{abstract}

Keywords: Burr Type XII Distribution, Maximum Likelihood Estimation, EM Algorithm, Censored Data

(c) 2014 Penerbit UTM Press. All rights reserved http://dx.doi.org/10.11113/mjfas.v10n2.58

\section{INTRODUCTION}

The word 'Burr' was introduced by [1] in 1942 when a few forms of cumulative distribution function were suggested to fit the data. Burr Type XII was used in many fields because of the potentiality in practical situations. In his guide to the Dagum distribution (2007), [2] stated that in economics, the Burr Type XII distribution is known as the Singh-Maddala distribution. Burr Type XII distribution has at least two unknown parameter. [3] derived the probability density function of a six-parameter generalized Burr Type XII distribution and obtained cumulative distribution function meanwhile [4] introduced properties of seven parameters Burr Type XII distribution.

In statistics, estimation process is very important to find the approximate value of unknown parameters. Several methods have been used to estimate the parameters of the Burr Type XII distribution such as Maximum Likelihood Estimation (MLE), Least Squares Estimation (LSE) and Bayesian Estimation. The parameter estimation process involves the presence of complete and censored data.

Nowadays, researchers used censored data to estimate the parameter. Different mechanisms can lead to different type of censored data such as right-censored, leftcensored and randomly censored. The usage of censored data gives the possibility to compute the estimation method to fit a model to censored data.
This paper aims to estimate the parameters $c$ and $k$ for 2-parameter and $c$, $\mathrm{k}$ and $s$ for 3-parameter of Burr Type XII distribution with complete and censored data using two methods which include MLE and EM algorithm approaches. Then, the estimated parameters from both methods were compared based on bias and mean square error (MSE).

\section{ESTIMATION OF PARAMETER IN BURR TYPE XII DISTRIBUTION}

\subsection{2-Parameter Burr Type XII distribution}

The probability density function ( $p d f$ ) of the standard Burr Type XII distribution for 2-parameter is written in the form of

$$
f(t ; c, k)=c k t^{c-1}\left(1+t^{c}\right)^{-(k+1)}
$$

and cumulative distribution function $(c d f)$

$$
F(t ; c, k)=1-\left(1+t^{c}\right)^{-k}
$$

for $t \geq 0$ with both $c$ and $k$ are shape parameters. 


\subsubsection{Maximum likelihood estimation (MLE)}

MLE is the most popular method of parameter estimation. The likelihood function of the censored data is given by

$$
L=\prod_{i=1}^{r} f\left(t_{i}\right) \prod_{j=r+1}^{n}\left[1-F\left(t_{j}\right)\right]=\prod_{i=1}^{r} \frac{c k t_{i}^{c-1}}{\left(1+t_{i}^{c}\right)^{k+1}} \prod_{j=r+1}^{n} \frac{1}{\left(1+t_{j}^{c}\right)^{k}}
$$

There are $r$ failures at times $t_{1}, t_{2}, \ldots, t_{r}$.

With $f(t)$ and $F(t)$ given by equations (1) and (2) respectively, the logarithm of the likelihood function becomes

$\ln L=r \ln c+r \ln k+(c-1) \sum_{i=1}^{r} \ln t_{i}-(k+1) \sum_{i=1}^{r} \ln \left(1+t_{i}{ }^{c}\right)-k \sum_{j=r+1}^{n} \ln \left(1+t_{j}{ }^{c}\right)$

Differentiate equation (4) with respect to $c$ and $k$ and equate each result to zero, then

$$
\begin{aligned}
& \frac{d \ln L}{d c}=\frac{r}{c}+\sum_{i=1}^{r} \ln t_{i}-(k+1) \sum_{i=1}^{r} \frac{t_{i}^{c} \ln t_{i}}{1+t_{i}^{c}}-k \sum_{j=r+1}^{n} \frac{t_{j}^{c} \ln t_{j}}{1+t_{j}^{c}}=0 \\
& \frac{d \ln L}{d k}=\frac{r}{k}-\sum_{i=1}^{r} \ln \left(1+t_{i}{ }^{c}\right)-\sum_{j=r+1}^{n} \ln \left(1+t_{j}{ }^{c}\right)=0
\end{aligned}
$$

Two equations are solved simultaneously to obtain the estimates of $c$ and $k$.

$$
\begin{gathered}
\sum_{i=1}^{r} \ln t_{i}=\sum_{i=1}^{r} \frac{t_{i}^{c} \ln t_{i}}{1+t_{i}^{c}}+\left[\left(\frac{r}{\sum_{i=1}^{r} \ln \left(1+t_{i}^{c}\right)+\sum_{j=r+1}^{n} \ln \left(1+t_{j}^{c}\right)}\right) \sum_{i=1}^{r} \frac{t_{i}^{c} \ln t_{i}}{1+t_{i}^{c}}\right] \\
+\left[\left(\frac{r}{\sum_{i=1}^{r} \ln \left(+t_{i}^{c}\right)+\sum_{j=r+1}^{n} \ln \left(1+t_{j}^{c}\right)}\right) \sum_{j=r+1}^{n} \frac{t_{j}^{c} \ln t_{j}}{1+t_{j}^{c}}\right]-\frac{r}{c}
\end{gathered}
$$$$
\hat{k}=\frac{r}{\sum_{i=1}^{r} \ln \left(1+t_{i}{ }^{c}\right)+\sum_{j=r+1}^{n} \ln \left(1+t_{j}{ }^{c}\right)}
$$

Equation (7) exhibits no explicit solutions to solve the equations analytically, and the maximization is performed through the mathematical approach that is the NewtonRaphson method to obtain the approximate solutions. According to [6], the definition is given by

$$
\begin{array}{ll}
S_{1}=\sum_{i=1}^{r} \ln t_{i} & S_{5}=\sum_{j=r+1}^{n} \ln \left(1+t_{j}{ }^{c}\right) \\
S_{2}=\sum_{i=1}^{r} \ln \left(1+t_{i}{ }^{c}\right) & S_{6}=\sum_{j=r+1}^{n} \frac{t_{j}{ }^{c} \ln t_{j}}{1+t_{j}{ }^{c}} \\
S_{3}=\sum_{i=1}^{r} \frac{t_{i}{ }^{c} \ln t_{i}}{1+t_{i}{ }^{c}} & S_{7}=\sum_{j=r+1}^{n} \frac{\left(t_{j}{ }^{c} \ln ^{2} t_{j}\right)\left(1+t_{j}{ }^{c}\right)-\left(t_{j}{ }^{c} \ln t_{j}\right)^{2}}{\left(1+t_{j}{ }^{c}\right)^{2}}
\end{array}
$$

$$
S_{4}=\sum_{i=1}^{r} \frac{\left(t_{i}^{c} \ln ^{2} t_{i}\right)\left(1+t_{i}^{c}\right)-\left(t_{i}^{c} \ln t_{i}\right)^{2}}{\left(1+t_{i}^{c}\right)^{2}}
$$

Then, equation (7) and (8) are solved as follows.

$\hat{c}_{i+1}=C_{i}+\left[\frac{\left(\frac{r}{C_{i}}+S_{1}-S_{3}-\frac{r}{\left(S_{2}+S_{5}\right.}\left(S_{3}+S_{6}\right)\right)}{\left(\frac{r}{c_{i}^{2}}+S_{4}-\frac{r}{\left(S_{2}+S_{5}\right)^{2} *\left(S_{3}+S_{6}\right)^{2}}+\frac{r}{\left(S_{2}+S_{5}\right)}\left(S_{4}+S_{7}\right)\right)}\right]$

$\hat{k}=\frac{r}{S_{2}+S_{5}}$

\subsubsection{EM Algorithm}

According to [5], let $y=\left(y_{1}{ }^{T}, \ldots, y_{n}{ }^{T}\right)^{T}$ denotes the observed data where $y_{i}=\left(d_{i}, \delta_{i}\right)^{T}$ and $\delta_{i}=0$ for censored data or 1 for failure (observed) data. $T_{i}$ is censored or uncensored at $d_{i}(i=1, \ldots, n)$. Then, the probability density function of 2-parameter Burr Type XII distribution when given $T>d_{j}$ is calculated as follows

$$
f\left(t \mid t>d_{j}\right)=\frac{f(t)}{1-F\left(d_{j}\right)}=c k\left(1+d_{j}^{c}\right)^{k} \frac{t^{c-1}}{\left(1+t^{c}\right)^{k+1}}, t>d_{j}
$$

From $f(t)$ in equation (1), the complete data log-likelihood function of the Burr Type XII distribution is expressed as

$$
\begin{aligned}
& \log \left(L_{c}(c, k)=\sum_{i=1}^{n} \log \left[f_{c}\left(t_{i} ; c, k\right)\right.\right. \\
& =n \log k+n \log c+(c-1) \sum_{i=1}^{n}\left(\log t_{i}\right)-(k+1) \sum_{i=1}^{n}\left[\log \left(1+t_{i}{ }^{c}\right)\right]
\end{aligned}
$$

The Q-function of 2-parameter Burr Type XII distribution of censored data is obtained as

$$
\begin{aligned}
& Q\left(\theta, \theta^{(m)}\right)=E_{\theta^{(m)}}\left[\log L_{c}(c, k)\right] \\
& =n \log k+n \log c+(c-1) \sum_{i=1}^{r} \log \left(d_{i}\right)-(k+1) \sum_{i=1}^{r} \log \left(1+d_{i}^{c}\right)+ \\
& (c-1) \sum_{j=r+1}^{n} E_{\theta^{m)}}\left(\log g_{j} \mid T_{j}>d_{j}\right)-(k+1) \sum_{j=+1}^{n} E_{\theta^{m}}\left[\log \left(+T_{j}^{c}\right) \mid T_{j}>d_{j}\right]
\end{aligned}
$$

Using numerical integral and apply Taylor series, equation (13) is solved as follows.

$$
\left.E_{\theta^{m}}\left(\log T_{j} \mid T_{j}>d_{j}\right)=\int_{d_{i}}^{\infty} \log t_{j}\right) c k\left(1+d_{j}^{c}\right)^{k} t_{j}^{c-1}\left(1+t_{j}^{c}\right)^{(-k-1)} d t_{j}
$$




$$
\begin{aligned}
& =c k\left(1+d_{j}^{c}\right)^{k} \int_{d_{i}}^{\infty} \frac{\left(\log _{j}\right)\left(t_{j}^{c-1}\right)}{\left(1+t_{j}^{c}\right)^{k+1}} d t_{j} \\
& E_{\theta^{(m)}}\left[\log \left(+T_{j}^{c}\right) \mid T_{j}>d_{j}\right] \cong E_{\theta^{(m)}}\left[\log \left(+T_{j}^{c}\right) \mid T_{j}>d_{j}\right]+ \\
& c-c^{(m)} E_{\theta^{(m)}}\left[\frac{T_{j}^{c}}{1+T_{j}^{c}} \log \left(T_{j}\right) \mid T_{j}>d_{j}\right]+ \\
& \frac{1}{2}\left(c-c^{(m)}\right)^{2} E_{\theta^{(m)}}\left[\frac{T_{j}^{c}}{\left(1+T_{j}^{c}\right)^{2}}(\log ())^{2} \mid T_{j}>d_{j}\right] \\
& E_{\theta^{(m)}}\left[\log \left(+T_{j}^{c}\right) \mid T_{j}>d_{j}\right]=\int_{d_{j}}^{\infty} \log \left(+t_{j}^{c}\right) \\
& c k\left(1+d_{j}{ }^{c}\right) t_{j}{ }^{c-1}\left(1+t_{j}{ }^{c}\right)^{(-k-1)} d t_{j} \\
& =c k\left(1+d_{j}{ }^{c}\right)^{k} \int_{d_{j}}^{\infty} \frac{\left[\log \left(1+t_{j}{ }^{c}\right)\right]\left(t_{j}{ }^{c-1}\right)}{\left(1+t_{j}{ }^{c}\right)^{k+1}} d t_{j} \\
& E_{\theta^{(m)}}\left[\frac{T_{j}^{c}}{1+T_{j}^{c}} \log \left(T_{j}\right) \mid T_{j}>d_{j}\right]=\int_{d_{j}}^{\infty} \frac{t_{j}^{c}}{1+t_{j}^{c}} \log \left(t_{j}\right) \\
& c k\left(1+d_{j}{ }^{c}\right) t_{j}^{c-1}\left(1+t_{j}{ }^{c}\right)^{(-k-1)} d t_{j} \\
& =c k\left(1+d_{j}{ }^{c}\right)^{k} \int_{d_{j}}^{\infty} \frac{\left[\log \left(t_{j}\right)\right]\left(t_{j}{ }^{2 c-1}\right)}{\left(1+t_{j}{ }^{c}\right)^{k+2}} d t_{j} \\
& E_{\theta^{(m)}}\left[\frac{T_{j}^{c}}{\left(1+T_{j}^{c}\right)^{2}}\left(\log \left(t_{i}\right)\right)^{2} \mid T_{j}>d_{j}\right]=\int_{d_{j}}^{\infty} \frac{t_{j}^{c}}{\left(1+t_{j}^{c}\right)^{2}} \\
& {\left[\log \left(\mathbb{t}_{j}\right)\right]^{2} c k\left(1+d_{j}^{c}\right) t_{j}^{c-1}\left(1+t_{j}^{c}\right)^{(-k-1)} d t_{j}} \\
& =c k\left(1+d_{j}{ }^{c}\right)^{k} \int_{d_{j}}^{\infty} \frac{\left[\log \left(t_{j}\right)\right]^{2}\left(t_{j}{ }^{2 c-1}\right)}{\left(1+t_{j}{ }^{c}\right)^{k+3}} d t_{j}
\end{aligned}
$$

\subsection{3-Parameter Burr Type XII distribution}

The probability density function ( $p d f$ ) of the standard Burr Type XII distribution for 3-parameter is written in the form of

$$
f(t ; c, k, s)=\frac{c k t^{c-1}}{s^{c}}\left[1+\left(\frac{t}{s}\right)^{c}\right]^{-(k+1)}
$$

and cumulative distribution function (cdf)

$$
F(t ; c, k, s)=1-\left[1+\left(\frac{t}{s}\right)^{c}\right]^{-k}
$$

for $t \geq 0$ with both $c$ and $k$ are shape parameters and $s$ is scale parameter

\subsubsection{Maximum Likelihood Estimation (MLE)} given by

The likelihood function for the censored data is

$$
L=\prod_{i=1}^{r} f\left(t_{i}\right) \prod_{j=r+1}^{n}\left[1-F\left(t_{j}\right)\right]=\prod_{i=1}^{r} \frac{c k\left(\frac{t_{i}}{s}\right)^{c-1}}{\left[1+\left(\frac{t_{i}}{s}\right)^{c}\right]^{k+1}} \prod_{j=r+1}^{n}\left[1+\left(\frac{t_{j}}{s}\right)^{c}\right]^{k}
$$

There are $r$ failures at times $t_{1}, t_{2}, \ldots, t_{r}$. Equations (12) and (13) become

$$
\begin{aligned}
& \ln L=r \ln c k-r \ln s+(c-1) \sum_{i=1}^{r} \ln \left(\frac{t_{i}}{s}\right)- \\
& \quad(k+1) \sum_{i=1}^{r} \ln \left[1+\left(\frac{t_{i}}{s}\right)^{c}\right]-k \sum_{j=r+1}^{n} \ln \left[1+\left(\frac{t_{j}}{s}\right)^{c}\right]
\end{aligned}
$$

Differentiate equation (17) with respect to $c, k$ and $s$ and equate each result to zero, the equation becomes

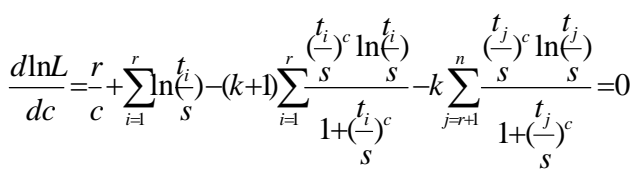

$$
\begin{aligned}
& \frac{d \ln L}{d k}=\frac{r}{k}-\sum_{i=1}^{r} \ln \left[1+\left(\frac{t_{i}}{s}\right)^{c}\right]-\sum_{j=r+1}^{n} \ln \left[1+\left(\frac{t_{j}}{s}\right)^{c}\right]=0 \\
& \frac{d \ln L}{d s}=-\frac{r}{s}+(c-1)\left(-\frac{1}{s}\right)-(k+1) \sum_{i=1}^{r} \frac{c\left(\frac{t_{i}}{s}\right)^{c-1}\left(\frac{t_{i}}{s^{c}}\right)}{\left[1+\left(\frac{t_{i}}{s}\right)^{c}\right]}-k \sum_{j=+1}^{n} \frac{c\left(\frac{t_{j}}{s}\right)^{c-1}\left(\frac{t_{j}}{s^{c}}\right)}{\left[1+\left(\frac{-}{s}\right)^{c}\right]} \\
& =\frac{r+c-1}{s}-(k+1) \sum_{i=1}^{r} \frac{c\left(\frac{t_{i}}{s}\right)^{c-1}\left(\frac{t_{i}}{s^{c}}\right)}{\left[1+\left(\frac{t_{i}}{s}\right)^{c}\right]}-k \sum_{j=+1}^{n} \frac{c\left(\frac{t_{j}}{s}\right)^{c-1}\left(\frac{t_{j}}{s^{c}}\right)}{\left[1+\left(\frac{t_{j}}{s}\right)^{c}\right]}
\end{aligned}
$$

Three equations are solved simultaneously to obtain the estimates of $c, k$ and $s$.

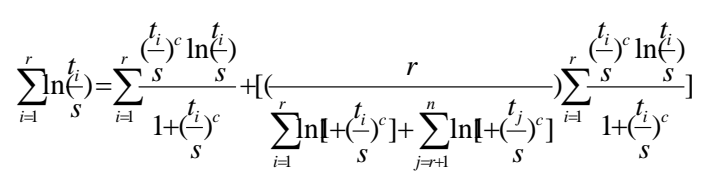

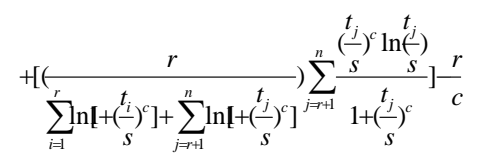

$$
\begin{aligned}
& k=\frac{r}{\sum_{i=1}^{r} \ln \left[1+\left(\frac{t_{i}}{s}\right)^{c}\right]+\sum_{j=r+1}^{n} \ln \left[1+\left(\frac{t_{j}}{s}\right)^{c}\right]} \\
& s=\frac{r+c-1}{(k+1) \sum_{i=1}^{r} \frac{c\left(\frac{t_{i}}{s}\right)^{c-1}\left(-\frac{t_{i}}{s^{c}}\right)}{\left[1+\left(\frac{t_{i}}{s}\right)^{c}\right]}+k \sum_{j=r+1}^{n} \frac{c\left(\frac{t_{j}}{s}\right)^{c-1}\left(-\frac{t_{j}}{s^{c}}\right)}{\left[1+\left(\frac{t_{j}}{s}\right)^{c}\right]}}
\end{aligned}
$$


The Newton-Raphson method is used to obtain the approximate solutions for equation (19) as follows.

$$
\begin{aligned}
& S_{1}=\sum_{i=1}^{r} \ln \left(\frac{t_{i}}{s}\right) \quad S_{5}=\sum_{j=r+1}^{n} \ln \left[1+\left(\frac{t_{j}}{s}\right)^{c}\right] \\
& S_{2}=\sum_{i=1}^{r} \ln \left[1+\left(\frac{t_{i}}{s}\right)^{c}\right] \quad S_{6}=\sum_{j=r+1}^{n} \frac{\left(\frac{t_{j}}{s}\right)^{c} \ln \left(\frac{t_{j}}{s}\right)}{\left[1+\left(\frac{t_{j}}{s}\right)^{c}\right]} \\
& S_{3}=\sum_{i=1}^{r} \frac{\left(\frac{t_{i}}{s}\right)^{c} \ln \left(\frac{t_{i}}{s}\right)}{1+\left(\frac{t_{i}}{s}\right)^{c}} \\
& S_{4}=\sum_{i=1}^{r} \frac{\left[\left(\frac{t_{i}}{s}\right)^{c} \ln ^{2}\left(\frac{t_{i}}{s}\right)\right]\left[1+\left(\frac{t_{i}}{s}\right)^{c}\right]-\left[\left(\frac{t_{i}}{s}\right)^{c} \ln \left(\frac{t_{i}}{s}\right)\right]^{2}}{\left[1+\left(\frac{t_{i}}{s}\right)^{c}\right]^{2}} \\
& S_{7}=\sum_{j=r+1}^{n} \frac{\left[\left(\frac{t_{j}}{s}\right)^{c} \ln ^{2}\left(\frac{t_{j}}{s}\right)\right]\left[1+\left(\frac{t_{j}}{s}\right)^{c}\right]-\left[\left(\frac{t_{j}}{s}\right)^{c} \ln \left(\frac{t_{j}}{s}\right)\right]^{2}}{\left[1+\left(\frac{t_{j}}{s}\right)^{c}\right]^{2}}
\end{aligned}
$$

Equation (21) and (22) are solved as follows

$$
\begin{aligned}
& \hat{c}_{i+1}=C_{i}+\left[\frac{\left(\frac{r}{C_{i}}+S_{1}-S_{3} \frac{n}{\left(S_{2}+S_{5}\right)}\left(S_{3}+S_{6}\right)\right)}{\left(\frac{r}{C_{i}^{2}}+S_{4}-\frac{n}{\left(S_{2}+S_{5}\right)^{2} *\left(S_{3}+S_{6}\right)^{2}}+\frac{r}{\left(S_{2}+S_{5}\right)}\left(S_{4}+S_{7}\right)\right)}\right] \\
& \hat{k}=\frac{r}{S_{2}+S_{5}}
\end{aligned}
$$

\subsubsection{EM Algorithm}

Let $y=\left(y_{1}^{T}, \ldots y_{n}^{T}\right)^{T}$ denote the observed data where $y_{i}=\left(d_{i}, \delta_{i}\right)^{T}$ and $\delta_{i}=0$ for censored data or 1 for failure (observe) data. $T_{i}$ is censored or uncensored at $d_{i}(i=1, \ldots, n)$. Then, the probability density function of 3-parameter Burr Type XII distribution when given $T>d_{j}$ is calculated as follows

$$
f\left(t \mid t>d_{j}\right)=\frac{f(t)}{1-F\left(d_{j}\right)}=\frac{c k}{s}\left[1+\left(\frac{d_{j}}{s}\right)^{c}\right]^{k} \frac{\left(\frac{t}{s}\right)^{c-1}}{\left[1+\left(\frac{d_{j}}{s}\right)^{c}\right]^{k+1}}, t>d_{j}
$$

$f(t)$ as given in equation (11) can be expressed as

$$
\begin{aligned}
& \log \left(L_{c}(c, k, s)=\sum_{i=1}^{n} \log \left[f_{c}\left(t_{i} ; c, k, s\right)\right.\right. \\
& =n \ln c k-n \ln s+(c-1) \sum_{i=1}^{n} \ln \left(\frac{t_{i}}{s}\right)-(k+1) \sum_{i=1}^{n} \ln \left[1+\left(\frac{t_{i}}{s}\right)^{c}\right]
\end{aligned}
$$

The Q-function of 3-parameter Burr Type XII distribution for multiple censored data is obtained as

$$
\begin{aligned}
& Q\left(\theta, \theta^{(m)}\right)=E_{\theta^{(m)}}\left[\log L_{c}(c, k, s)\right] \\
& =n \log c k-n \log s+(c-1) \sum_{i=1}^{r} \log \left(\frac{d_{i}}{S}\right)-(k+1) \sum_{i=1}^{r} \log \left(1+\left(\frac{d_{i}}{s}\right)^{c}\right)+ \\
& (c-1) \sum_{j=+1}^{n} E_{\theta^{(m)}}\left[\log _{S}\left(\mid T_{j}>d_{j}\right]-(k+1) \sum_{j=+1}^{n} E_{\theta^{(m)}}\left[\log \left(T_{j}\left(\frac{T_{j}}{{ }^{c}}\right) \mid T_{j}>d_{j}\right]\right.\right.
\end{aligned}
$$

Equation (28) is solved using numerical integral and applies Taylor series as follows.

$$
\begin{aligned}
& E_{\theta^{(m)}}\left[\log \left(\frac{T_{j}}{S}\right) \mid T_{j}>d{ }_{j}\right]=\int_{d_{i}}^{\infty} \log \left(\frac{t_{j}}{S}\right) \\
& c k\left[1+\left(\frac{d_{j}}{s}\right)^{c}\right]^{k}\left(\frac{t_{j}}{s}\right)^{c-1}\left[1+\left(\frac{t_{j}}{s}\right)^{c}\right]^{(-k-1)} d t_{j} \\
& =c k\left[1+\left(\frac{d_{j}}{s}\right)^{c}\right]^{k} \int_{d_{i}}^{\infty} \frac{\left[\log \left(\frac{t_{j}}{s}\right)\right]\left[\left(\frac{t_{j}}{s}\right)^{c-1}\right]}{\left[1+\left(\frac{t_{j}}{s}\right)^{c}\right]^{k+1}} d t_{j} \\
& E_{\theta^{m}}\left[\log \left(+\left(\frac{T_{j}}{S}\right)^{c}\right) \mid T_{j}>d_{j}\right] \cong E_{\theta^{m}}\left[\log \left(+\left(\frac{T_{j}}{S}\right)^{c}\right) \mid T_{j}>d_{j}\right]+ \\
& c-c^{(m)} E_{\theta^{(m)}}\left[\frac{\left(\frac{T_{j}}{S}\right)^{c}}{1+\left(\frac{T_{j}}{S}\right)^{c}} \log \left(\frac{T_{j}}{S}\right) \mid T_{j}>d_{j}\right]+
\end{aligned}
$$

$$
\frac{1}{2}\left(c-c^{(m)}\right)^{2} E_{\theta^{(m)}}\left[\frac{\left(\frac{T_{j}}{S}\right)^{c}}{\left(1+\left(\frac{T_{j}}{S}\right)^{c}\right)^{2}}\left(\log \left(_{S}\left(\frac{i}{S}\right)\right)^{2} \mid T_{j}>d_{j}\right]\right.
$$

$E_{\theta^{(m)}}\left[\log \left(+\left(\frac{T_{j}}{s}\right)^{c}\right) \mid T_{j}>d_{j}\right]=\int_{d_{j}}^{\infty} \log \left[\mathbb{l}+\left(\frac{t_{j}}{s}\right)^{c}\right]$

$$
\begin{aligned}
& c k\left[1+\left(\frac{d_{j}}{s}\right)^{c}\right]\left(\frac{t_{j}}{s}\right)^{c-1}\left[1+\left(\frac{t_{j}}{s}\right)^{c}\right]^{(-k-1)} d t_{j} \\
& =c k\left[1+\left(\frac{d_{j}}{s}\right)^{c}\right] \int_{d_{j}}^{\infty} \frac{\left[\log \left(1+\left(\frac{t_{j}}{s}\right)^{c}\right)\right]\left[\left(\frac{t_{j}}{s}\right)^{c-1}\right]}{\left[1+\left(\frac{t_{j}}{s}\right)^{c}\right]^{k+1}} d t_{j}
\end{aligned}
$$

$E_{\theta^{(m)}}\left[\frac{\left(\frac{T_{j}}{S}\right)^{c}}{1+\left(\frac{T_{j}}{S}\right)^{c}} \log \left(\frac{T_{j}}{S}\right) \mid T_{j}>d_{j}\right]=\int_{d_{j}\left[1+\left(\frac{t_{j}}{S}\right)^{c}\right]}^{\infty} \frac{\left(\frac{t_{j}}{S}\right)^{c}}{t_{S}}\left(_{\frac{j}{j}}\right)$ 


$$
\begin{gathered}
c k\left[1+\left(\frac{d_{j}}{s}\right)^{c}\right]\left(\frac{t_{j}}{s}\right)^{c-1}\left[1+\left(\frac{t_{j}}{s}\right)^{c}\right]^{(-k-1)} d t_{j} \\
=c k\left[1+\left(\frac{d_{j}}{s}\right)^{c}\right] \int_{d_{j}}^{\infty} \frac{\left[\log \left(\frac{t_{j}}{s}\right)\right]\left[\left(\frac{t_{j}}{s}\right)^{2 c-1}\right]}{\left[1+\left(\frac{t_{j}}{s}\right)^{c}\right]^{k+2}} d t_{j} \\
\left.\left.E_{\theta^{(m)}}\left[\frac{\left(\frac{T_{j}}{s}\right)^{c}}{\left(1+\left(\frac{T_{j}}{s}\right)^{c}\right)^{2}}\left(\log \left(\frac{T_{i}}{s}\right)\right)^{2} \mid T_{j}>d_{j}\right]=\int_{d_{j}}^{\infty} \frac{\left(1+\left(\frac{t_{j}}{s}\right)^{c}\right.}{s}\right)^{c}\right]^{2} \\
\left.\log \left(\frac{t_{j}}{s}\right)\right]^{2} \times \\
=c k\left[1+\left(\frac{d+}{s}\right)^{c}\right]\left(\frac{t_{j}}{s}\right)^{c-1}\left[1+\left(\frac{t_{j}}{s}\right)^{c}\right]^{(-k-1)} d t_{j} \\
\left.\left.\left.c\left(\frac{d}{s}\right)^{c}\right] \int_{d_{j}}^{\infty} \frac{\left[\log \left(\frac{t_{j}}{s}\right)\right]^{2}\left[\left(\frac{t_{j}}{s}\right)^{2 c-1}\right]}{t_{j}}\right)^{c}\right]^{k+3} t_{j}
\end{gathered}
$$

\subsection{Efficiency and accuracy}

The bias is calculated by the difference between the expected value of an estimator and the true value of the estimator in order to ascertain the accuracy of the estimators in the model. Let $\hat{\theta}$ is the estimator of the parameter, and then biasedness is calculated as

$$
\operatorname{Bias}(\hat{\theta})=E(\hat{\theta})-\theta
$$

Mean square error (MSE) is a way of measurement through the average of the square error.

$$
\begin{aligned}
M S E & =E\left[(\hat{\theta}-\theta)^{2}\right] \\
M S E & =\operatorname{Var}(\hat{\theta})+[\operatorname{Bias}(\hat{\theta})]^{2}
\end{aligned}
$$

It provides better quality measurement for the estimator since it accesses on both the variances and bias term.

\subsection{SIMULATIONS AND RESULTS}

1000 numbers of complete sample sizes were generated and the curve showed that the simulated data were well fitted to the estimated 2-parameter Burr Type XII survival function in Figure (a). Figure (b) and (c) show that the complete simulated data undergone some amount of censoring and each of them contains $10 \%$ and $30 \%$ of the censored data respectively.

The Burr Type XII distribution will be written as $\operatorname{Burr}_{\mathrm{XII}}(c, k)$ for 2-parameter and $\operatorname{Burr}_{\mathrm{XII}}(c, k, s)$ for 3parameter. The estimated parameter of 2-parameter Burr Type XII distribution is investigated by varying the $k$ values in Table 1 and $c$ values in Table 2 . The discussion of the estimated parameter will be given to $\operatorname{Burr}_{\mathrm{XII}}(1,2)$ from Table 1 since the explanations were the same for other parameter values. The sample size is standardized to 200 and the result for the MLE and EM algorithm showed the estimated parameter for $c$ and $k$ are approximately close to the true parameter of 1 and 2 for uncensored data. With respect to the bias and MSE of parameter $c$ and $k$, the EM algorithm outperforms the MLE with bias is -0.0764 for $c$ parameter and 0.0317 for the $k$ parameter; with MSE is 0.0058 and 0.0010 for $c$ and $k$ parameter respectively which is smaller than the bias and MSE of MLE with bias 0.1241 and -0.0702 for $c$ and $k$ parameter respectively and MSE is 0.0154 for $c$ and 0.0049 for $k$ parameter.

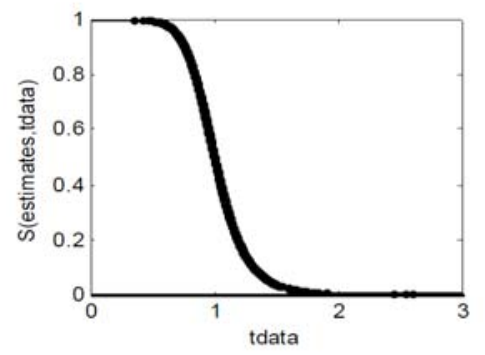

(a)

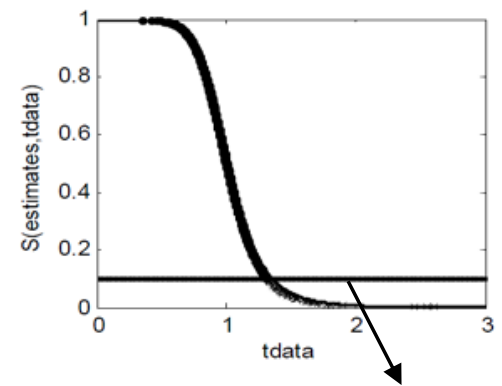

(b)

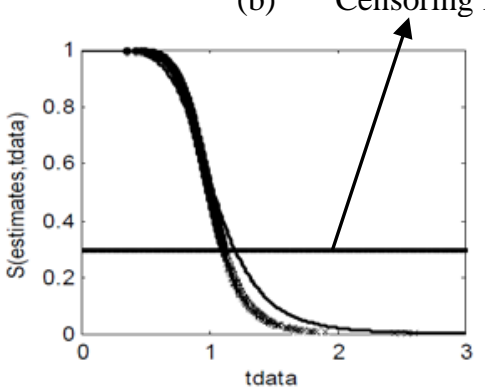

(c)

Figure 1 Curve fitting comparison of 2-parameter Burr Type XII with (a) uncensored, (b) $10 \%$ censoring and (c) 30\% censoring

In the presence of $30 \%$ censoring level, the accuracy of $\hat{c}$ and $\hat{k}$ dropped with the value 1.1816 and 1.7132 respectively. Table 1 showed that when the percentage of incomplete data (censored) is increased, the value of estimated parameter $c$ and $k$ is also increased. However, the EM algorithm still gives the smaller values of bias and MSE than the MLE. This is also same for several values of $c$ and $k$.

The estimated parameter of Burr Type XII distribution will be discussed only to the example of Burr XII 
$(2,5,1)$ from Table 4 since the explanations were the same for other parameter values by varying the $s$ values in Table $3, k$ values in Table 4 and $c$ values in Table 5 for 3 parameter. The estimated parameter for $c, k$ and $s$ for complete data are approximately close to the true parameter of 2, 5 and 1 respectively. The bias of the EM algorithm is smaller than the MLE method with 0.0194 for $c$ parameter, 0.0526 for $k$ parameter and 0.0025 for the $s$ parameter and the MSEs $0.0061,0.0045$ and 0.0001 for $c, k$ and $s$ parameters respectively. The estimated values of $s$ parameter for $10 \%$ and $30 \%$ censoring level are very far away from the true value for both approaches. This is because $s$ is the scale parameter and since we used random censoring, the scale parameter change and different from the true value. However, the EM algorithm still outperforms MLE with respect to the bias and MSE (see in Appendix A).

\subsection{CONCLUSION}

MLE and EM algorithm approaches are presented in this research to estimate the 2- and 3-parameter Burr Type XII distribution of complete and censored data. The estimated parameters of the Burr Type XII distribution are away from the true value as the percentage of censoring level increase. The model with the less censoring is performed better than the great censoring. Based on bias and MSE of 2- and 3-parameter Burr Type XII distribution for both approaches, the estimated parameters using EM algorithm gives the smaller value than the estimated parameter using MLE estimates for complete and censored data. EM algorithm has the advantage and is stable in numerical computation because of its robustness against the initial value. Lastly, for further research, EM algorithm can be used to estimate the parameter for another type of Burr distribution and compare with other estimation approaches such as Bayesian method.

\section{ACKNOWLEDGMENTS}

The authors would like to thank the Malaysian Ministry of Higher Education and Universiti Teknologi Malaysia for their financial funding through FRGS grant 4F004.

\section{REFERENCES}

[1] I. W. Burr, Ann. Math. Stat. 13 (1942) 215.

[2] C. A. Kleiber, Guide to the Dagum Distributions. Center of Business and Economics (WWZ). (2007)

[3] A.K. Olapade, Electron. J. Stat. (2008)

[4] A. H. El-Bassiouny and N. F. Abdo, Computational Methods in Science and Technology, 16(2) (2010) 127.

[5] F. K. Wang, and Y. F. Cheng, Qual. Reliab. Eng. Int. 26 (2009) 615.

[6] F. K. Wang, J. B. Keats and W. J. Zimmer, Microelectron. Reliab. 36(1) (1996) 359. 


\section{Appendix A}

Table 1 Comparison of the estimators, bias, MSE (parentheses) and negative log-likelihood for multiple data sets of 2-parameter Burr Type XII distribution with true value of $c=3$ and $k=2,5$, 8

\begin{tabular}{|c|c|c|c|c|c|c|c|c|c|c|c|}
\hline \multirow[t]{2}{*}{$k$} & \multirow[t]{2}{*}{$\mathbf{C L}$} & \multicolumn{2}{|c|}{ MIE } & \multicolumn{2}{|c|}{ Bias (MSE) } & \multirow[t]{2}{*}{$-\ln L$} & \multicolumn{2}{|r|}{ EM } & \multicolumn{2}{|c|}{ Bias (MSE) } & \multirow[t]{2}{*}{$-\ln L$} \\
\hline & & $c$ & $k$ & $c$ & $k$ & & $c$ & $k$ & $c$ & $k$ & \\
\hline & $0 \%$ & 2.9911 & 2.0565 & -0.0089 & 0.0565 & 72.3900 & 2.9989 & 2.0495 & -0.0011 & 0.0495 & 72.3923 \\
\hline & & & & $(0.0001)$ & $(0.0032)$ & & & & $(0.0000)$ & $(0.0025)$ & \\
\hline \multirow[t]{6}{*}{2} & $10 \%$ & 3.0324 & 1.9862 & 0.0324 & -0.0138 & 72.7297 & 3.0166 & 2.0807 & 0.0166 & 0.0807 & 72.9342 \\
\hline & & & & $(0.0010)$ & $(0.0002)$ & & & & $(0.0003)$ & $(0.0065)$ & \\
\hline & $30 \%$ & 3.0648 & 1.8877 & 0.0648 & -0.1123 & 67.1335 & 2.9794 & 2.0925 & -0.0206 & 0.0925 & 68.0573 \\
\hline & & & & $(0.0042)$ & $(0.0126)$ & & & & $(0.0004)$ & $(0.0086)$ & \\
\hline & $0 \%$ & 3.0695 & 5.3724 & 0.0695 & 0.3724 & -32.8630 & 3.0655 & 5.1685 & 0.0655 & 0.1685 & -32.7243 \\
\hline & & & & $(0.0048)$ & $(0.1387)$ & & & & $(0.0043)$ & $(0.0284)$ & \\
\hline \multirow[t]{6}{*}{5} & $10 \%$ & 3.2108 & 5.4182 & 0.2108 & 0.4182 & -28.5975 & 3.0995 & 5.2493 & 0.0995 & 0.2493 & -28.3740 \\
\hline & & & & $(0.0444)$ & $(0.1749)$ & & & & $(0.0099)$ & $(0.0622)$ & \\
\hline & $30 \%$ & 3.2707 & 4.8173 & 0.2707 & -0.1827 & -8.5840 & 3.1085 & 5.2698 & 0.1085 & 0.2698 & -6.7639 \\
\hline & & & & $(0.0733)$ & $(0.0334)$ & & & & $(0.0118)$ & $(0.0728)$ & \\
\hline & $0 \%$ & 3.0457 & 7.8380 & 0.0457 & -0.1620 & -65.0026 & 3.0156 & 8.0526 & 0.0156 & 0.0526 & -64.8149 \\
\hline & & & & $(0.0021)$ & $(0.0262)$ & & & & $(0.0002)$ & $(0.0028)$ & \\
\hline \multirow[t]{4}{*}{8} & $10 \%$ & 3.0562 & 7.7978 & 0.0562 & -0.2022 & -55.2219 & 3.0330 & 7.8265 & 0.0330 & -0.1735 & -55.1928 \\
\hline & & & & $(0.0032)$ & $(0.0409)$ & & & & $(0.0011)$ & $(0.0301)$ & \\
\hline & $30 \%$ & 3.1769 & 7.8810 & 0.1769 & -0.1190 & -33.5429 & 3.0586 & 8.1907 & 0.0586 & 0.1907 & -32.6455 \\
\hline & & & & $(0.0313)$ & $(0.0142)$ & & & & $(0.0034)$ & $(0.0364)$ & \\
\hline
\end{tabular}

Table 2 Comparison of the estimators, bias, MSE (parentheses) and negative log-likelihood for multiple data sets of 2-parameter Burr Type XII distribution with true value of $c=1,2,4$ and $\mathrm{k}=2$

\begin{tabular}{|c|c|c|c|c|c|c|c|c|c|c|c|}
\hline \multirow[t]{2}{*}{$c$} & \multirow[t]{2}{*}{ CL } & \multicolumn{2}{|c|}{ MILE } & \multicolumn{2}{|c|}{ Bias (MSE) } & \multirow[t]{2}{*}{$-\ln L$} & \multicolumn{2}{|r|}{ EM } & \multicolumn{2}{|c|}{ Bias (MSE) } & \multirow[t]{2}{*}{$-\ln L$} \\
\hline & & $c$ & $k$ & $c$ & $k$ & & $c$ & $k$ & $c$ & $k$ & \\
\hline & $0 \%$ & 1.1241 & 1.9298 & 0.1241 & -0.0702 & 170.2058 & 0.9236 & 2.0317 & -0.0764 & 0.0317 & 176.1415 \\
\hline & & & & $(0.0154)$ & $(0.0049)$ & & & & $(0.0058)$ & $(0.0010)$ & \\
\hline \multirow[t]{6}{*}{1} & $10 \%$ & 1.1251 & 1.8185 & 0.1251 & -0.1815 & 164.1454 & 1.1032 & 1.8489 & 0.1032 & -0.1511 & 164.2259 \\
\hline & & & & $(0.0157)$ & $(0.0329)$ & & & & $(0.0107)$ & $(0.0228)$ & \\
\hline & $30 \%$ & 1.1830 & 1.5363 & 0.1830 & -0.4637 & 152.5279 & 1.1816 & 1.7132 & 0.1816 & -0.2868 & 153.3904 \\
\hline & & & & $(0.0335)$ & $(0.2150)$ & & & & $(0.0330)$ & $(0.0823)$ & \\
\hline & $0 \%$ & 1.9928 & 2.0311 & -0.0072 & 0.0311 & 120.5805 & 1.9991 & 2.0070 & -0.0009 & 0.0070 & 120.5964 \\
\hline & & & & $(0.0001)$ & $(0.0010)$ & & & & $(0.0000)$ & $(0.0000)$ & \\
\hline \multirow[t]{6}{*}{2} & $10 \%$ & 2.0184 & 1.9062 & 0.0184 & -0.0938 & 120.0149 & 1.9915 & 1.9266 & -0.0085 & -0.0734 & 120.0529 \\
\hline & & & & $(0.0003)$ & $(0.0088)$ & & & & $(0.0001)$ & $(0.0054)$ & \\
\hline & $30 \%$ & 2.0573 & 1.6798 & 0.0573 & -0.3202 & 111.8820 & 2.0433 & 1.8722 & 0.0433 & -0.1278 & 112.7460 \\
\hline & & & & $(0.0033)$ & $(0.1025)$ & & & & $(0.0019)$ & $(0.0163)$ & \\
\hline & $0 \%$ & 4.0774 & 1.9177 & 0.0774 & -0.0823 & 34.5685 & 3.9688 & 2.0516 & -0.0312 & 0.0516 & 35.1391 \\
\hline & & & & $(0.0060)$ & $(0.0068)$ & & & & $(0.0010)$ & $(0.0027)$ & \\
\hline \multirow[t]{4}{*}{4} & $10 \%$ & 4.0592 & 1.8471 & 0.0592 & -0.1529 & 39.3504 & 4.0420 & 1.9394 & 0.0420 & -0.0606 & 39.5695 \\
\hline & & & & $(0.0035)$ & $(0.0234)$ & & & & $(0.0018)$ & $(0.0037)$ & \\
\hline & $30 \%$ & 4.2276 & 1.8175 & 0.2276 & -0.1825 & 34.6019 & 4.1415 & 2.1559 & 0.1415 & 0.1559 & 36.8425 \\
\hline & & & & $(0.0518)$ & $(0.0333)$ & & & & $(0.0200)$ & $(0.0243)$ & \\
\hline
\end{tabular}


Table 3 Comparison of the estimators, bias, MSE (parentheses) and negative log-likelihood for multiple data sets of 3parameter Burr Type XII distribution with true value of $c=2, k=3$ and $s=1,3$

\begin{tabular}{|c|c|c|c|c|c|c|c|c|c|c|c|c|c|c|c|}
\hline \multirow[t]{2}{*}{$s$} & \multirow[t]{2}{*}{ CL } & \multicolumn{3}{|c|}{ MLE } & \multicolumn{3}{|c|}{ Bias (MSE) } & \multirow[t]{2}{*}{$-\ln L$} & \multicolumn{3}{|c|}{ MLE } & \multicolumn{3}{|c|}{ Bias (MSE) } & \multirow[t]{2}{*}{$-\ln L$} \\
\hline & & $c$ & $k$ & $s$ & $c$ & $k$ & $s$ & & $c$ & $k$ & $s$ & $c$ & $k$ & $s$ & \\
\hline & $0 \%$ & 2.0278 & 2.9791 & 0.9938 & 0.0278 & -0.0209 & -0.0062 & 59.7459 & 2.0167 & 2.9990 & 0.9967 & 0.0167 & -0.0010 & -0.0033 & 58.6142 \\
\hline & & & & & $(0.0008)$ & $(0.0004)$ & $(0.0000)$ & & & & & $(0.0003)$ & $(0.0000)$ & $(0.0000)$ & \\
\hline \multirow[t]{6}{*}{1} & $10 \%$ & 2.0331 & 2.9300 & 3.3024 & 0.0331 & -0.0700 & 2.3024 & -146.6664 & 1.9731 & 3.0431 & 3.0786 & -0.0269 & 0.0431 & 2.0786 & -160.8956 \\
\hline & & & & & $(0.0011)$ & $(0.0049)$ & $(5.3010)$ & & & & & $(0.0007)$ & $(0.0019)$ & $(4.3206)$ & \\
\hline & $30 \%$ & 2.0488 & 2.5393 & 12.9523 & 0.0488 & -0.4607 & 11.9523 & -103.2675 & 2.0431 & 2.6270 & 12.8095 & 0.0431 & -0.3730 & 11.8095 & -110.2365 \\
\hline & & & & & $(0.0024)$ & $(0.2122)$ & $(142.8575)$ & & & & & $(0.0019)$ & $(0.1391)$ & (139.4643) & \\
\hline & $0 \%$ & 2.0402 & 3.0488 & 3.0074 & 0.0402 & 0.0488 & 0.0074 & -168.1272 & 2.0346 & 3.0051 & 2.9945 & 0.0346 & 0.0051 & -0.0055 & -166.4025 \\
\hline & & & & & $(0.0016)$ & $(0.0024)$ & $(0.0001)$ & & & & & $(0.0012)$ & $(0.0000)$ & $(0.0000)$ & \\
\hline \multirow[t]{4}{*}{3} & $10 \%$ & 2.0603 & 2.9432 & 4.2020 & 0.0603 & -0.0568 & 1.2020 & -240.1818 & 2.0514 & 3.0343 & 4.0423 & 0.0514 & 0.0343 & 1.0423 & -233.6244 \\
\hline & & & & & $(0.0036)$ & $(0.0032)$ & (1.4448) & & & & & $(0.0026)$ & $(0.0012)$ & (1.0864) & \\
\hline & $30 \%$ & 2.1626 & 2.7475 & 6.7819 & 0.1626 & -0.2525 & 3.7819 & -223.6393 & 2.0851 & 3.1046 & 6.5125 & 0.0851 & 0.1046 & 3.5125 & -241.6338 \\
\hline & & & & & $(0.0264)$ & $(0.0638)$ & (14.3028) & & & & & $(0.0072)$ & $(0.0109)$ & (12.3377) & \\
\hline
\end{tabular}

Table 4 Comparison of the estimators, bias, MSE (parentheses) and negative log-likelihood for multiple data sets of 3parameter Burr Type XII distribution with true value of $c=2, k=1,5$ and $s=1$

\begin{tabular}{|c|c|c|c|c|c|c|c|c|c|c|c|c|c|c|c|}
\hline \multirow[t]{2}{*}{$k$} & \multirow[t]{2}{*}{ CL } & \multicolumn{3}{|c|}{ MLE } & \multicolumn{3}{|c|}{ Bias (MSE) } & \multirow[t]{2}{*}{$-\ln L$} & \multicolumn{3}{|c|}{ MLE } & \multicolumn{3}{|c|}{ Bias (MSE) } & \multirow[t]{2}{*}{$-\ln L$} \\
\hline & & $c$ & $k$ & $s$ & $c$ & $k$ & $s$ & & $c$ & $k$ & $s$ & $c$ & $k$ & $s$ & \\
\hline & $0 \%$ & 1.8754 & 1.1811 & 0.7012 & -0.1246 & 0.1811 & -0.2988 & 450.0444 & 2.0779 & 1.0672 & 1.0112 & 0.0779 & 0.0672 & 0.0112 & 268.2439 \\
\hline & & & & & $(0.0155)$ & $(0.0328)$ & $(0.0893)$ & & & & & $(0.0061)$ & $(0.0045)$ & $(0.0001)$ & \\
\hline \multirow[t]{6}{*}{1} & $10 \%$ & 2.2237 & 0.7437 & 16.2757 & 0.2237 & $\begin{array}{l}-0.2563 \\
(0.06577\end{array}$ & 15.2757 & 8.6902 & 1.9155 & 1.1748 & 16.2427 & $\begin{array}{l}-0.0845 \\
(0.071)\end{array}$ & 0.1748 & 15.2427 & -193.4323 \\
\hline & & & & & & & & & & & & & & & \\
\hline & $30 \%$ & 2.4473 & 0.6476 & 44.7391 & 0.4473 & -0.3524 & 43.7391 & 155.4795 & 2.1003 & 1.2531 & 44.3236 & 0.1003 & 0.2531 & 43.3236 & -95.4526 \\
\hline & & & & & $(0.2001)$ & $(0.1242)$ & (1913.1089) & & & & & $(0.0101)$ & $(0.0641)$ & (1876.9343) & \\
\hline & $0 \%$ & 2.0403 & 5.1358 & 1.0106 & 0.0403 & 0.1358 & 0.0106 & -21.9258 & 2.0194 & 5.0526 & 1.0025 & 0.0194 & 0.0526 & 0.0025 & -18.7267 \\
\hline & & & & & $(0.0016)$ & $(0.0184)$ & $(0.0001)$ & & & & & $(0.0004)$ & $(0.0028)$ & $(0.0000)$ & \\
\hline \multirow[t]{4}{*}{5} & $10 \%$ & 2.0685 & 5.0572 & 3.1564 & 0.0685 & 0.0572 & 2.1564 & -187.3184 & 2.0245 & 5.0613 & 3.0281 & 0.0245 & 0.0613 & 2.0281 & -197.0788 \\
\hline & & & & & $(0.0047)$ & $(0.0033)$ & (4.6501) & & & & & $(0.0006)$ & $(0.0038)$ & (4.1132) & \\
\hline & $30 \%$ & 2.1601 & 4.8090 & 8.1730 & 0.1601 & -0.1910 & 7.1730 & -112.5038 & 1.8489 & 5.1537 & 8.0241 & -0.1511 & 0.1537 & 7.0241 & -232.9054 \\
\hline & & & & & $(0.0256)$ & $(0.0365)$ & (51.4519) & & & & & $(0.0228)$ & $(0.0236)$ & $(49.3380)$ & \\
\hline
\end{tabular}

Table 5 Comparison of the estimators, bias, MSE (parentheses) and negative log-likelihood for multiple data sets of 3parameter Burr Type XII distribution with true value of $c=0.5,2, k=2$ and $s=2$

\begin{tabular}{|c|c|c|c|c|c|c|c|c|c|c|c|c|c|c|c|}
\hline \multirow[t]{2}{*}{$c$} & \multirow[t]{2}{*}{ CL } & \multicolumn{3}{|c|}{ MLE } & \multicolumn{3}{|c|}{ Bias (MSE) } & \multirow[t]{2}{*}{$-\ln L$} & \multicolumn{3}{|c|}{ MLE } & \multicolumn{3}{|c|}{ Bias (MSE) } & \multirow[t]{2}{*}{$-\ln L$} \\
\hline & & $c$ & $k$ & $s$ & $c$ & $k$ & $s$ & & $c$ & $k$ & $s$ & $c$ & $k$ & $s$ & \\
\hline & $0 \%$ & 0.5099 & 1.9041 & 2.0245 & 0.0099 & -0.0959 & 0.0245 & -29.8771 & 0.4966 & 2.0135 & 2.1089 & -0.0034 & 0.0135 & 0.1089 & -46.3002 \\
\hline & & & & & $(0.0001)$ & $(0.0092)$ & $(0.0006)$ & & & & & $(0.0000)$ & $(0.0002)$ & $(0.0119)$ & \\
\hline \multirow[t]{6}{*}{0.5} & $10 \%$ & 0.5213 & 1.8274 & 6.7165 & 0.0213 & -0.1726 & 4.7165 & -417.8265 & 0.4835 & 2.1217 & 6.3874 & -0.0165 & 0.1217 & 4.3874 & -411.1649 \\
\hline & & & & & $(0.0005)$ & $(0.0298)$ & (22.2454) & & & & & $(0.0003)$ & $(0.0148)$ & (19.2493) & \\
\hline & $30 \%$ & 0.5289 & 1.7233 & 11.1714 & 0.0289 & -0.2767 & 9.1714 & -423.4693 & 0.4723 & 2.2153 & 10.982 & -0.0277 & 0.2153 & 8.9820 & -432.5536 \\
\hline & & & & & $(0.0008)$ & $(0.0766)$ & $(84.1146)$ & & & & & $(0.0008)$ & $(0.0464)$ & $(80.6763)$ & \\
\hline & $0 \%$ & 1.9076 & 2.0659 & 1.9957 & -0.0924 & 0.0659 & -0.0043 & -16.5046 & 2.0207 & 2.0595 & 2.0042 & 0.0207 & 0.0595 & 0.0042 & -17.6473 \\
\hline & & & & & $(0.0085)$ & $(0.0043)$ & $(0.0000)$ & & & & & $(0.0004)$ & $(0.0035)$ & $(0.0000)$ & \\
\hline \multirow[t]{4}{*}{2} & $10 \%$ & 1.8888 & 2.2623 & 3.9560 & -0.1112 & 0.2623 & 1.9560 & -202.1237 & 1.9005 & 1.9227 & 3.8156 & -0.0995 & -0.0773 & 1.8156 & -177.0412 \\
\hline & & & & & $(0.0124)$ & $(0.0688)$ & $(3.8259)$ & & & & & $(0.0099)$ & $(0.0060)$ & (3.2964) & \\
\hline & $30 \%$ & 1.8342 & 2.2730 & 7.8225 & -0.1658 & 0.2730 & 5.8225 & -232.4619 & 1.8374 & 2.0933 & 7.6836 & -0.1626 & 0.0933 & 5.6836 & -220.9551 \\
\hline & & & & & $(0.0275)$ & $(0.0745)$ & $(33.9015)$ & & & & & $(0.0264)$ & $(0.0087)$ & (32.3033) & \\
\hline
\end{tabular}

